Canadian

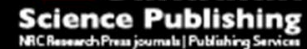

Canadian Journal of Chemistry Revue canadienne de chimie

\title{
Molecular Description of Carbon Graphite and Crystal Cubic Carbon Structures
}

\begin{tabular}{|c|c|}
\hline Journal: & Canadian Journal of Chemistry \\
\hline Manuscript ID & cjc-2017-0083.R1 \\
\hline Manuscript Type: & Article \\
\hline Date Submitted by the Author: & 01-Mar-2017 \\
\hline Complete List of Authors: & $\begin{array}{l}\text { Baig, A. Q.; COMSATS Insitute of Information Technology, Attock, } \\
\text { Pakistan, Mathematics } \\
\text { Imran, Muhammad; United Arab Emirates University, Mathematical } \\
\text { Sciences; National University of Sciences and Technology } \\
\text { Khalid, Waqas; COMSATS Institute of Information Technology - Attock } \\
\text { Naeem, Muhammad; COMSATS Institute of Information Technology }\end{array}$ \\
\hline \multicolumn{2}{|l|}{$\begin{array}{l}\text { Please Select from this Special } \\
\text { Issues list if applicable: }\end{array}$} \\
\hline Keyword: & $\begin{array}{l}\text { Molecular Descriptors, Carbon Graphite, Crystal Cubic Carbon, Atom-bond } \\
\text { connectivity index, geometric-arithmetic index }\end{array}$ \\
\hline
\end{tabular}




\title{
Molecular Description of Carbon Graphite and Crystal Cubic Carbon Structures
}

\author{
Abdul Qudair Baig ${ }^{1}$, Muhammad Imran $^{2,3}$, Waqas Khalid ${ }^{1}$, Muhammad Naeem $^{4}$ \\ ${ }^{1}$ Department of Mathematics, \\ COMSATS Institute of Information Technology, \\ Attock, Pakistan \\ ${ }^{2}$ Department of Mathematical Sciences, \\ United Arab Emirates University, \\ P.O. Box 15551, \\ Al Ain, United Arab Emirates \\ ${ }^{3}$ School of Natural Sciences(SNS), \\ National University of Sciences and Technology (NUST), \\ Sector H-12, Islamabad, Pakistan \\ ${ }^{4}$ Department of Mathematics, \\ COMSATS Institute of Information Technology, \\ Sahiwal, Pakistan \\ E-mail: $\left\{\right.$ aqbaig $1^{1}$, imrandhab ${ }^{2,3}$, waqas.khalid $38^{1}$, naeempkn $\left.{ }^{4}\right\} @$ gmail.com \\ 2,3 Muhammad Imran
}




\begin{abstract}
Graph theory plays a vital role in modeling and designing any chemical structure or chemical network. Chemical graph theory helps in understanding about the molecular structural properties of a molecular graph. The molecular graph is a graph consists of atoms called vertices and the chemical bond between atoms called edges.

In this article, we study the chemical graphs of carbon graphite and crystal structure of cubic carbon. Moreover, we compute and give closed formulas of degree based additive topological indices mainly first and second Zagreb index, general Randić, atom-bond connectivity index, geometric arithmetic index, fourth atom-bond connectivity and fifth geometric arithmetic index of carbon graphite denoted by $C G(m, n)$ for $t$-levels and crystal structure cubic carbon for $n$-levels.
\end{abstract}

Keywords: Molecular Descriptors, Carbon Graphite, Crystal Cubic Carbon, Atom bond connectivity index, Geometric arithmetic index, General Randić index, $A B C_{4}, G A_{5}$. 


\section{Introduction}

Graph theory is proficient for modeling and designing of chemical structures and complex networks. The chemical graph theory applies graph theory to mathematical modeling of molecular phenomena, which is helpful for the study of molecular structure. This theory contributes a prominent role in the field of chemical sciences. Chemical compounds have a variety of applications in chemical graph theory, drug design, etc. The manipulation and examination of chemical structural information is made conceivable by using molecular descriptors. A great variety of topological indices are studied and used in theoretical chemistry, pharmaceutical researchers. A chemical structure can be represented by using graph theory, where vertices denotes atoms and edges denotes molecular bond. A topological index is a numeric number which indicates some useful information about molecular structure. It is the numerical invariants of a molecular graph and are useful to correlate with their bioactivity and physio-chemical properties. Researchers have found topological index to be powerful and useful tool in the description of molecular structure. Some applications related to topological indices of molecular graphs are given $\mathrm{in}^{2,3,9,12,13,18,19,23,26,27,28}$ and also the references therein. The researchers work in the field of chemical graph theory about the topological applications of carbon nanocones, extremal pentagonal chains, tree like polyphenylene, spiro hexagonal systems, polyphenylene dendrimer nanostars, nanostructures, and polyomino chains in in $^{1,4-6,25}$. These chemical applications are motivated us to study topological descriptors and compute for some new chemical graphs.

There are certain chemical compounds that are useful for the survival of living things. Carbon, oxygen, hydrogen and nitrogen are the main elements that helps in the production of cells in the living things. Carbon is an essential element for human life. It is useful in the formation of proteins, carbohydrates and nucleic acids. It is vital for the growth of plants in the form of carbon dioxide. The carbon atoms can bond together in various ways, called allotropes of carbon. The well known forms are graphite and diamond. Recently, many new forms have been discovered including nanotubes, buckminsterfullerene and sheets, crystal cubic structure, etc. The applications of different allotropes of carbon are discussed in detail in ${ }^{22,29}$.

Let $G=(V, E)$ be a graph where $V$ be the vertex set and $E$ be the edge set of $G$. The degree $\operatorname{deg}(v)$ of $v$ is the number of edges of $G$ incident with $v$. The length of a shortest path in a graph $G$ is a distance $d(u, v)$ between $u$ and $v$. A graph can be represented by a polynomial, a numerical value or by matrix form. There are certain types of topological indices mainly eccentric based, degree based and distance based indices etc. In this article, we deal with degree based topological indices.

One of the first and oldest degree based index is introduced by Milan Randic ${ }^{24}$ in 1975 and is defined 
below.

$$
R_{-\frac{1}{2}}(G)=\sum_{u v \in E(G)} \frac{1}{\sqrt{d_{u} d_{v}}}
$$

In 1988, Bollobás et al. ${ }^{2}$ and Amic et al. ${ }^{1}$ proposed the general Randić index independently. For more details about Randić index, its properties and important results ${ }^{10,20,21}$. The general Randić index is defined as

$$
R_{\alpha}(G)=\sum_{u v \in E(G)}\left(d_{u} d_{v}\right)^{\alpha}
$$

Among degree based topological indices, atom bond connectivity index of vital importance and introduced by Estrada et al. ${ }^{11}$ and is defined as

$$
A B C(G)=\sum_{u v \in E(G)} \sqrt{\frac{d_{u}+d_{v}-2}{d_{u} d_{v}}}
$$

where $d_{u}$ is the degree of vertex $u$.

One of the important degree based topological index is the first Zagreb index. It was introduced in 1972 by ${ }^{17}$. Later on, second Zagreb index is introduced $b^{16}$. Both first and second Zagreb index is formulated as

$$
\begin{aligned}
M_{1}(G) & =\sum_{u v \in E(G)}\left(d_{u}+d_{v}\right) \\
M_{2}(G) & =\sum_{u v \in E(G)}\left(d_{u} d_{v}\right)
\end{aligned}
$$

A well known topological index fourth version of atom bond connectivity index $A B C_{4}$ of a graph $G$ is introduced by Ghorbhani et al. ${ }^{14}$ and is defined as

$$
A B C_{4}(G)=\sum_{u v \in E(G)} \sqrt{\frac{S_{u}+S_{v}-2}{S_{u} S_{v}}}
$$

where $S_{u}=\sum_{u v \in E(G)} d_{v}$ and $S_{v}=\sum_{u v \in E(G)} d_{u}$.

The geometric arithmetic index $G A$ of a graph $G$ is introduced by Vukičević et al. ${ }^{30}$ and is defined as

$$
G A(G)=\sum_{u v \in E(G)} \frac{2 \sqrt{d_{u} d_{v}}}{d_{u}+d_{v}}
$$

Another well known topological descriptor fifth version of geometric arithmetic index $G A_{5}$ of a graph $G$ is introduced by Graovoc et al. ${ }^{15}$ and is defined as

$$
G A_{5}(G)=\sum_{u v \in E(G)} \frac{2 \sqrt{S_{u} S_{v}}}{S_{u}+S_{v}}
$$




\section{Main results}

In this section, additive topological indices mainly atom bond connectivity index, geometric arithmetic index, fourth atom bond connectivity index $A B C_{4}$, fifth geometric arithmetic index $G A_{5}$, first and second Zagreb index of carbon graphite and crystal structure cubic carbon are computed. Also, we compute general Randić index for $\alpha=\left\{1,-1, \frac{1}{2},-\frac{1}{2}\right\}$ only. Moreover, closed formulas are derived which are helpful for the study analysis of properties of molecular structures of $C G(m, n)$ and $C C C(n)$.

\subsection{Carbon Graphite}

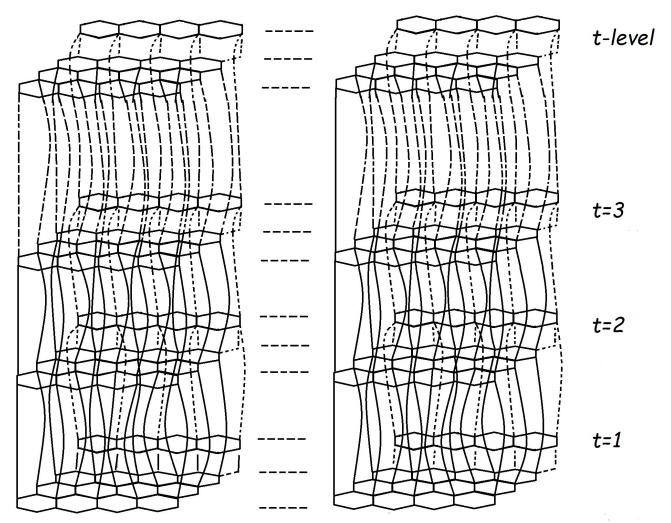

Fig. 1: Carbon graphite $C G(m, n)$ t-levels.

Graphite is an allotrope of carbon. The chemical graph of carbon graphite $C G(m, n)$ consist of a hexagon shapes. The structure of carbon graphite consists of hexagons in the form of layers one after the other and between these layers there is a weak bond. The molecular graph of carbon graphite $C G(m, n)$ for $t$-levels is depicted in Fig. 1. The cardinality of vertices and edges in $C G(m, n)$ are $2 t(m n+m+n)$ and $4 m n t+3 m t+2 n t-m n-m-t$ respectively. A single layer in carbon graphite depicts the graphene layer. Carbon graphite consists of multiple layers of graphene. The $t$ is the levels that depicts graphene layers in carbon graphite, $m$ is the number of rows and $n$ is the number of columns in each layer with $m$ is taken as $n$ copies of hexagons in a row and $n$ is taken as $m$ copies of hexagons in column. In Fig. 2, the levels of carbon graphite is $t=3, m=3$ and $n=4$, where $m=3$ is taken as $n=4$ copies of hexagons in a row in each level $t=3$ and $n=4$ is taken as $m=3$ copies of hexagons in column in each level.

In $C G(m, n)$, the 2 degree vertices are $2(n t+m+1)$, the 3 degree vertices are $2 m n+2 m t-2 m+2 t-4$ and 4 degree vertices are $2(m n-1)(t-1)$. The edge set of $C G(m, n)$ is divided into six partitions based on the degree of end vertices. The first edge partition contains 4 edges $u v$, where $\operatorname{deg}(u)=\operatorname{deg}(v)=2$. The second edge partition contains $4(n+t-1)$ edges $u v$, where $\operatorname{deg}(u)=2$ and $\operatorname{deg}(v)=3$. The third edge partition contains $4(n t+m-n-t)$ edges $u v$, where $\operatorname{deg}(u)=2$ and $\operatorname{deg}(v)=4$. The fourth edge 
partition contains $4 m+4 t-10$ edges $u v$, where $\operatorname{deg}(u)=\operatorname{deg}(v)=3$. The fifth edge partition contains $6 m n+6 m t-14 m-4 n-6 t+12$ edges $u v$, where $\operatorname{deg}(u)=3$ and $\operatorname{deg}(v)=4$. The sixth edge partition contains $(4 m n-3 m-2 n+1) t-7 m n+5 m+4 n-2$ edges $u v$, where $\operatorname{deg}(u)=\operatorname{deg}(v)=4$. The Table 1 shows such an edge partition of $C G(m, n)$ for $m, n>1$ and $t \geq 3$.

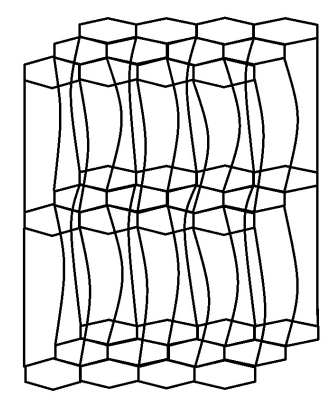

Fig. 2: Carbon graphite graph for $(3,4)$ and $t=3$.

\begin{tabular}{|c|c|}
\hline$\left(d_{u}, d_{v}\right)$ & frequency \\
\hline$(2,2)$ & 4 \\
\hline$(2,3)$ & $4(n+t-1)$ \\
\hline$(2,4)$ & $4(n t+m-n-t)$ \\
\hline$(3,3)$ & $4 m+4 t-10$ \\
\hline$(3,4)$ & $6 m n+6 m t-14 m-4 n-6 t+12$ \\
\hline$(4,4)$ & $(4 m n-3 m-2 n+1) t-7 m n+5 m+4 n-2$ \\
\hline
\end{tabular}

Table 1: Edge partition of $C G(m, n)$ based on degree sum of end vertices of each edge.

In the coming theorem, we compute atom bond connectivity index of $C G(m, n)$.

Theorem 2.1. Consider the graph of carbon graphite $C G(m, n)$ with $m, n>1$ and $t \geq 3$, then its atom bond connectivity index is equal to

$$
\begin{aligned}
& A B C(G)=m\left(2 \sqrt{2}+\frac{5 \sqrt{6}}{4}-\frac{7 \sqrt{15}}{3}+\frac{8}{3}\right)+n\left(\sqrt{6}-\frac{2 \sqrt{15}}{3}\right)+m n\left(\frac{-7 \sqrt{6}}{4}+\sqrt{15}\right)+t\left(2 \sqrt{2} n+\frac{(4 m n-3 m-2 n+1) \sqrt{6}}{4}+\right. \\
& \left.(m-1) \sqrt{15}+\frac{8}{3}\right)-\frac{\sqrt{6}}{2}+2 \sqrt{15}-\frac{20}{3} .
\end{aligned}
$$

Proof. Let $G \cong C G(m, n)$ be a graph of carbon graphite. The edge partition of $C G(m, n)$ based on degrees of end vertices of each edge with their frequencies is given in Table 1 . The atom bond connectivity index can be calculated by using Table 1 in the following equation.

$$
A B C(G)=\sum_{u v \in E(G)} \sqrt{\frac{d_{u}+d_{v}-2}{d_{u} d_{v}}}
$$


$A B C(G)=(4) \sqrt{\frac{2+2-2}{2 \times 2}}+4(n+t-1) \sqrt{\frac{2+3-2}{2 \times 3}}+4(n t+m-n-t) \sqrt{\frac{2+4-2}{2 \times 4}}+(4 m+4 t-10) \sqrt{\frac{3+3-2}{3 \times 3}}+$ $(6 m n+6 m t-14 m-4 n-6 t+12) \sqrt{\frac{3+4-2}{3 \times 4}}+((4 m n-3 m-2 n+1) t-7 m n+5 m+4 n-2) \sqrt{\frac{4+4-2}{4 \times 4}}$

After an easy calculation, we get

$$
\begin{aligned}
& A B C(G)=m\left(2 \sqrt{2}+\frac{5 \sqrt{6}}{4}-\frac{7 \sqrt{15}}{3}+\frac{8}{3}\right)+n\left(\sqrt{6}-\frac{2 \sqrt{15}}{3}\right)+m n\left(\frac{-7 \sqrt{6}}{4}+\sqrt{15}\right)+t\left(2 \sqrt{2} n+\frac{(4 m n-3 m-2 n+1) \sqrt{6}}{4}+\right. \\
& \left.(m-1) \sqrt{15}+\frac{8}{3}\right)-\frac{\sqrt{6}}{2}+2 \sqrt{15}-\frac{20}{3}
\end{aligned}
$$

In the next theorem, we compute Randić index $R_{\alpha}(G)$ of carbon graphite $C G(m, n)$.

Theorem 2.2. Consider the graph of carbon graphite $C G(m, n)$ with $m, n>1$ and $t \geq 3$, then its general Randić index is equal to

$$
R_{\alpha} C G(m, n)= \begin{cases}-20 m+8 n-40 m n+t(64 m n+ & \\ 24 m-28)+14, & \text { if } \alpha=1, \\ \frac{1}{4}\left(\frac{13}{36} m+\frac{1}{3} n+\frac{1}{4} m n+t\left(\frac{5 m}{4}\right.\right. & \\ \left.\left.+\frac{3 n}{2}+m n+\frac{25}{36}\right)+\frac{7}{18}\right), & \\ m\left(\sqrt{2}-\frac{7}{\sqrt{3}}+\frac{31}{12}\right)-n\left(\sqrt{2}+\frac{2}{\sqrt{3}}\right. & \\ \left.-\frac{4}{\sqrt{6}}-1\right)+m n\left(\sqrt{3}-\frac{7}{4}\right) & \\ \left.+(m-1) \sqrt{3}+\frac{4}{\sqrt{6}}+\frac{19}{12}\right)+2 \sqrt{3} & \\ -\frac{4}{\sqrt{6}}-\frac{11}{6}, & \\ m(8 \sqrt{2}-28 \sqrt{3}+32)-n(8 \sqrt{2}+8 \sqrt{3} & \\ -4 \sqrt{6}-16)+m n(12 \sqrt{3}-28) & \\ +t(16 m n-12 m-8 n+8(n-1) \sqrt{2} & \\ +12(m-1) \sqrt{3}+4 \sqrt{6}+16) & \\ +24 \sqrt{3}-4 \sqrt{6}-30 . & \\ & \end{cases}
$$

Proof. Let $G \cong C G(m, n)$ be a graph of carbon graphite. The above result can be proved by using Table 1 in the following computation. The general Randić index for $\alpha=1$.

$$
R_{1}(G)=\sum_{u v \in E(G)}\left(d_{u} \times d_{v}\right)
$$

$R_{1}(G)=(4)(2 \times 2)+4(n+t-1)(2 \times 3)+4(n t+m-n-t)(2 \times 4)+(4 m+4 t-10)(3 \times 3)+(6 m n+$ $6 m t-14 m-4 n-6 t+12)(3 \times 4)+((4 m n-3 m-2 n+1) t-7 m n+5 m+4 n-2)(4 \times 4)$ 
$R_{1}(G)=-20 m+8 n-40 m n+t(64 m n+24 m-28)+14$

For $\alpha=-1$, the formula of Randić index takes the following form.

$$
R_{-1}(G)=\sum_{u v \in E(G)} \frac{1}{\left(d_{u} \times d_{v}\right)}
$$

$R_{-1}(G)=(4) \frac{1}{(2 \times 2)}+4(n+t-1) \frac{1}{(2 \times 3)}+4(n t+m-n-t) \frac{1}{(2 \times 4)}+(4 m+4 t-10) \frac{1}{(3 \times 3)}+(6 m n+6 m t-$ $14 m-4 n-6 t+12) \frac{1}{(3 \times 4)}+((4 m n-3 m-2 n+1) t-7 m n+5 m+4 n-2) \frac{1}{(4 \times 4)}$

$R_{-1}(G)=\frac{1}{4}\left(\frac{13}{36} m+\frac{1}{3} n+\frac{1}{4} m n+t\left(\frac{5 m}{4}+\frac{3 n}{2}+m n+\frac{25}{36}\right)+\frac{7}{18}\right)$

For $\alpha=-\frac{1}{2}$, the formula of Randić index takes the following form.

$$
\begin{gathered}
R_{-\frac{1}{2}}(G)=\sum_{u v \in E(G)} \frac{1}{\sqrt{\left(d_{u} \times d_{v}\right)}} \\
R_{-\frac{1}{2}}(G)=(4) \frac{1}{\sqrt{(2 \times 2)}}+4(n+t-1) \frac{1}{\sqrt{(2 \times 3)}}+4(n t+m-n-t) \frac{1}{\sqrt{(2 \times 4)}}+(4 m+4 t-10) \frac{1}{\sqrt{(3 \times 3)}}+(6 m n+ \\
6 m t-14 m-4 n-6 t+12) \frac{1}{\sqrt{(3 \times 4)}}+((4 m n-3 m-2 n+1) t-7 m n+5 m+4 n-2) \frac{1}{\sqrt{(4 \times 4)}} \\
R_{-\frac{1}{2}}(G)=m\left(\sqrt{2}-\frac{7}{\sqrt{3}}+\frac{31}{12}\right)-n\left(\sqrt{2}+\frac{2}{\sqrt{3}}-\frac{4}{\sqrt{6}}-1\right)+m n\left(\sqrt{3}-\frac{7}{4}\right)+t\left(m n-\frac{3 m}{4}-\frac{n}{2}+(n-\right. \\
\left.1) \sqrt{2}+(m-1) \sqrt{3}+\frac{4}{\sqrt{6}}+\frac{19}{12}\right)+2 \sqrt{3}-\frac{4}{\sqrt{6}}-\frac{11}{6}
\end{gathered}
$$

For $\alpha=\frac{1}{2}$, the formula of Randić index takes the following form.

$$
R_{\frac{1}{2}}(G)=\sum_{u v \in E(G)} \sqrt{\left(d_{u} \times d_{v}\right)}
$$

$R_{\frac{1}{2}}(G)=(4) \sqrt{(2 \times 2)}+4(n+t-1) \sqrt{(2 \times 3)}+4(n t+m-n-t) \sqrt{(2 \times 4)}+(4 m+4 t-10) \sqrt{(3 \times 3)}+$ $(6 m n+6 m t-14 m-4 n-6 t+12) \sqrt{(3 \times 4)}+((4 m n-3 m-2 n+1) t-7 m n+5 m+4 n-2) \sqrt{(4 \times 4)}$

$R_{\frac{1}{2}}(G)=m(8 \sqrt{2}-28 \sqrt{3}+32)-n(8 \sqrt{2}+8 \sqrt{3}-4 \sqrt{6}-16)+m n(12 \sqrt{3}-28)+t(16 m n-12 m-8 n+$ $8(n-1) \sqrt{2}+12(m-1) \sqrt{3}+4 \sqrt{6}+16)+24 \sqrt{3}-4 \sqrt{6}-30$.

A close formula of geometric arithmetic index $G A$ of $C G(m, n)$ is computed in the following theorem.

Theorem 2.3. Consider the graph of carbon graphite $C G(m, n)$, for $m, n>1$ and $t \geq 3$, then its geometric arithmetic index is equal to 
$G A(G)=m\left(\frac{8 \sqrt{2}}{3}-8 \sqrt{3}+9\right)-n\left(\frac{8 \sqrt{2}}{3}+\frac{16 \sqrt{3}}{7}-\frac{8 \sqrt{6}}{5}-4\right)+m n\left(\frac{24 \sqrt{3}}{7}-7\right)+t(4 m n-3 m-2 n+$ $\left.\frac{8(n-1) \sqrt{2}}{3}+\frac{24(m-1) \sqrt{3}}{7}+\frac{8 \sqrt{6}}{5}+5\right)+\frac{48 \sqrt{3}}{7}-\frac{8 \sqrt{6}}{5}-8$.

Proof. Let $G \cong C G(m, n)$ be a graph of carbon graphite. The above result can be proved by using Table 1 in the following computation. The geometric arithmetic index is computed as below:

$$
G A(G)=\sum_{u v \in E(G)} \frac{2 \sqrt{d_{u} d_{v}}}{d_{u}+d_{v}}
$$

$G A(G)=(4) \frac{2 \sqrt{2 \times 2}}{2+2}+4(n+t-1) \frac{2 \sqrt{2 \times 3}}{2+3}+4(n t+m-n-t) \frac{2 \sqrt{2 \times 4}}{2+4}+(4 m+4 t-10) \frac{2 \sqrt{3 \times 3}}{3+3}+(6 m n+6 m t-$ $14 m-4 n-6 t+12) \frac{2 \sqrt{3 \times 4}}{3+4}+((4 m n-3 m-2 n+1) t-7 m n+5 m+4 n-2) \frac{2 \sqrt{4 \times 4}}{4+4}$

After an easy calculation, we get

$G A(G)=m\left(\frac{8 \sqrt{2}}{3}-8 \sqrt{3}+9\right)-n\left(\frac{8 \sqrt{2}}{3}+\frac{16 \sqrt{3}}{7}-\frac{8 \sqrt{6}}{5}-4\right)+m n\left(\frac{24 \sqrt{3}}{7}-7\right)+t(4 m n-3 m-2 n+$ $\left.\frac{8(n-1) \sqrt{2}}{3}+\frac{24(m-1) \sqrt{3}}{7}+\frac{8 \sqrt{6}}{5}+5\right)+\frac{48 \sqrt{3}}{7}-\frac{8 \sqrt{6}}{5}-8$.

In the next theorem, we compute first and second Zagreb index of $C G(m, n)$.

Theorem 2.4. Consider the graph $G \cong C G(m, n)$, for $m, n>1$, then its first and second Zagreb index is equal to

$$
\begin{aligned}
& M_{1}(G)=-10 m-14 m n+t(32 m n+18 m-40 n+34)+4 . \\
& M_{2}(G)=-20 m+8 n-40 m n+t(64 m n+24 m-28)+14 .
\end{aligned}
$$

Proof. Let $G \cong C G(m, n)$ be a graph of carbon graphite. The above result can be proved by using Table 1 in the following computation. The first Zagreb index is computed as below:

$$
M_{1}(G)=\sum_{u v \in E(G)}\left(d_{u}+d_{v}\right) .
$$

$M_{1}(G)=(4)(2+2)+4(n+t-1)(2+3)+4(n t+m-n-t)(2+4)+(4 m+4 t-10)(3+3)+(6 m n+$ $6 m t-14 m-4 n-6 t+12)(3+4)+((4 m n-3 m-2 n+1) t-7 m n+5 m+4 n-2)(4+4)$

$M_{1}(G)=-10 m-14 m n+t(32 m n+18 m-40 n+34)+4$.

The second Zagreb index is computed as below:

$$
M_{2}(G)=\sum_{u v \in E(G)}\left(d_{u} d_{v}\right) .
$$

$M_{2}(G)=(4)(2 \times 2)+4(n+t-1)(2 \times 3)+4(n t+m-n-t)(2 \times 4)+(4 m+4 t-10)(3 \times 3)+(6 m n+$ $6 m t-14 m-4 n-6 t+12)(3 \times 4)+((4 m n-3 n-2 n+1) t-7 m n+5 m+4 n-2)(4 \times 4)$ 
$M_{2}(G)=-20 m+8 n-40 m n+t(64 m n+24 m-28)+14$.

The Table 2 shows the edge partition based on the degree sum of end vertices of each edge. We compute fourth atom bond connectivity index and fifth geometric arithmetic index by using Table 2 .

\begin{tabular}{|c|c|c|c|}
\hline$\left(S_{u}, S_{v}\right)$ & Number of edges & $\left(S_{u}, S_{v}\right)$ & Number of edges \\
\hline$(5,5)$ & 2 & $(8,12)$ & $4(n t-2 n-2 t+4)$ \\
\hline$(5,6)$ & 2 & $(8,13)$ & $2(n-1)$ \\
\hline$(5,8)$ & 6 & $(9,10)$ & 2 \\
\hline$(6,8)$ & $4(n-2)$ & $(9,13)$ & 2 \\
\hline$(6,10)$ & 2 & $(10,10)$ & $4 m-6$ \\
\hline$(7,7)$ & 4 & $(10,12)$ & $4 m-6$ \\
\hline$(7,8)$ & $2(t-2)$ & $(10,13)$ & $2(n-1)$ \\
\hline$(7,9)$ & 2 & $(11,12)$ & $2(n-1)$ \\
\hline$(7,10)$ & $2 t$ & $(11,16)$ & $6 m n+6 m t-22 m-6 n-12 t+36$ \\
\hline$(7,11)$ & 4 & $(12,13)$ & $4 n t-10 n-4 t+10$ \\
\hline$(7,12)$ & $4(t-2)$ & $(12,16)$ & $2 m n+2 m t-6 m-4 n-2 t+8$ \\
\hline$(8,8)$ & 2 & $(13,16)$ & \\
\hline$(8,10)$ & $4(m+t-4)$ & $(16,16)$ & $4 m n t-5 m t-6 n t-9 m n+11 m+14 n+7 t-16$ \\
\hline$(8,11)$ & $4(n-2)$ & & \\
\hline
\end{tabular}

Table 2: Edge partition of $C G(m, n)$ based on degree sum of end vertices of each edge.

A close formula of fourth atom bond connectivity index $A B C_{4}$ of $C G(m, n)$ is computed in the following theorem.

Theorem 2.5. Consider the graph of carbon graphite $C G(m, n)$ with $m, n>2$ and $t \geq 3$, then its fourth atom bond connectivity index is equal to

$$
\begin{aligned}
& A B C_{4}(G)=m\left(\frac{6 \sqrt{2}}{5}+\frac{4}{\sqrt{5}}+\frac{4}{\sqrt{6}}+\frac{11 \sqrt{30}}{16}-\frac{9 \sqrt{39}}{26}-\frac{11 \sqrt{897}}{39}+\frac{2 \sqrt{2730}}{65}\right)+n\left(\frac{5 \sqrt{11}}{22}+\sqrt{\frac{19}{26}}+\frac{7 \sqrt{30}}{8}-\frac{3 \sqrt{39}}{13}+\frac{\sqrt{77}}{11}-\right. \\
& \left.\frac{5 \sqrt{78}}{12}+\frac{\sqrt{374}}{11}-\frac{\sqrt{897}}{13}+2\right)-m n\left(\frac{9 \sqrt{30}}{16}-\frac{3 \sqrt{39}}{26}-\frac{\sqrt{897}}{13}\right)+t\left((n-2) \sqrt{3}+\frac{4}{\sqrt{5}}+\sqrt{\frac{13}{14}}+\frac{(4 m n-5 m-6 n+7) \sqrt{30}}{16}+\right. \\
& \left.\frac{3(m-1) \sqrt{39}}{26}+\frac{\sqrt{42}}{7}+\frac{(n-1) \sqrt{78}}{6}+\frac{2 \sqrt{357}}{21}+\frac{(m-2) \sqrt{897}}{13}+\frac{3 \sqrt{2730}}{65}\right)-\frac{\sqrt{2}}{3}-\frac{6 \sqrt{3}}{7}-\frac{16}{\sqrt{5}}-\sqrt{6}-\frac{5 \sqrt{11}}{22}-2 \sqrt{\frac{13}{14}}+\frac{\sqrt{14}}{4}- \\
& \sqrt{\frac{19}{26}}-\frac{4 \sqrt{30}}{5}+\frac{6 \sqrt{39}}{13}+\frac{4 \sqrt{65}}{39}+\frac{16}{\sqrt{77}}-\frac{\sqrt{77}}{11}+\frac{5 \sqrt{78}}{12}+\frac{3 \sqrt{110}}{10}+\frac{\sqrt{170}}{15}+\frac{\sqrt{210}}{15}-\frac{4 \sqrt{357}}{21}-\frac{2 \sqrt{374}}{11}+\frac{6 \sqrt{897}}{13}-\frac{2 \sqrt{2730}}{13}-4 .
\end{aligned}
$$

Proof. Let $G \cong C G(m, n)$ be a graph of carbon graphite. The above result can be proved by using Table 2 in the following computation. The fourth atom bond connectivity index is computed as below:

$$
A B C_{4}(G)=\sum_{u v \in E(G)} \sqrt{\frac{S_{u}+S_{v}-2}{S_{u} S_{v}}}
$$

$$
\begin{aligned}
& A B C_{4}(G)=(2) \sqrt{\frac{5+5-2}{5 \times 5}}+(2) \sqrt{\frac{5+6-2}{5 \times 6}}+(6) \sqrt{\frac{5+8-2}{5 \times 8}}+4(n-2) \sqrt{\frac{6+8-2}{6 \times 8}}+(2) \sqrt{\frac{6+10-2}{6 \times 10}}+(4) \sqrt{\frac{7+7-2}{7 \times 7}}+ \\
& 2(t-2) \sqrt{\frac{7+8-2}{7 \times 8}}+(2) \sqrt{\frac{7+9-2}{7 \times 9}}+(2 t) \sqrt{\frac{7+10-2}{7 \times 10}}+(4) \sqrt{\frac{7+11-2}{7 \times 11}}+4(t-2) \sqrt{\frac{7+12-2}{7 \times 12}}+(2) \sqrt{\frac{8+8-2}{8 \times 8}}+4(m+ \\
& t-4) \sqrt{\frac{8+10-2}{8 \times 10}}+4(n-2) \sqrt{\frac{8+11-2}{8 \times 11}}+4(n t-2 n-2 t+4) \sqrt{\frac{8+12-2}{8 \times 12}}+2(n-1) \sqrt{\frac{8+13-2}{8 \times 13}}+(2) \sqrt{\frac{9+10-2}{9 \times 10}}+
\end{aligned}
$$


(2) $\sqrt{\frac{9+13-2}{9 \times 13}}+(4 m-6) \sqrt{\frac{10+10-2}{10 \times 10}}+(4 m-6) \sqrt{\frac{10+12-2}{10 \times 12}}+(4 m+6 t-20) \sqrt{\frac{10+13-2}{10 \times 13}}+2(n-1) \sqrt{\frac{11+12-2}{11 \times 12}}+$ $2(n-1) \sqrt{\frac{11+16-2}{11 \times 16}}+(6 m n+6 m t-22 m-6 n-12 t+36) \sqrt{\frac{12+13-2}{12 \times 13}}+(4 n t-10 n-4 t+10) \sqrt{\frac{12+16-2}{12 \times 16}}+$ $(2 m n+2 m t-6 m-4 n-2 t+8) \sqrt{\frac{13+16-2}{13 \times 16}}+(4 m n t-5 m t-6 n t-9 m n+11 m+14 n+7 t-16) \sqrt{\frac{16+16-2}{16 \times 16}}$

After an easy calculation, we get

$A B C_{4}(G)=m\left(\frac{6 \sqrt{2}}{5}+\frac{4}{\sqrt{5}}+\frac{4}{\sqrt{6}}+\frac{11 \sqrt{30}}{16}-\frac{9 \sqrt{39}}{26}-\frac{11 \sqrt{897}}{39}+\frac{2 \sqrt{2730}}{65}\right)+n\left(\frac{5 \sqrt{11}}{22}+\sqrt{\frac{19}{26}}+\frac{7 \sqrt{30}}{8}-\frac{3 \sqrt{39}}{13}+\frac{\sqrt{77}}{11}-\right.$
$\left.\frac{5 \sqrt{78}}{12}+\frac{\sqrt{374}}{11}-\frac{\sqrt{897}}{13}+2\right)-m n\left(\frac{9 \sqrt{30}}{16}-\frac{3 \sqrt{39}}{26}-\frac{\sqrt{897}}{13}\right)+t\left((n-2) \sqrt{3}+\frac{4}{\sqrt{5}}+\sqrt{\frac{13}{14}}+\frac{(4 m n-5 m-6 n+7) \sqrt{30}}{16}+\right.$
$\left.\frac{3(m-1) \sqrt{39}}{26}+\frac{\sqrt{42}}{7}+\frac{(n-1) \sqrt{78}}{6}+\frac{2 \sqrt{357}}{21}+\frac{(m-2) \sqrt{897}}{13}+\frac{3 \sqrt{2730}}{65}\right)-\frac{\sqrt{2}}{3}-\frac{6 \sqrt{3}}{7}-\frac{16}{\sqrt{5}}-\sqrt{6}-\frac{5 \sqrt{11}}{22}-2 \sqrt{\frac{13}{14}}+\frac{\sqrt{14}}{4}-$
$\sqrt{\frac{19}{26}}-\frac{4 \sqrt{30}}{5}+\frac{6 \sqrt{39}}{13}+\frac{4 \sqrt{65}}{39}+\frac{16}{\sqrt{77}}-\frac{\sqrt{77}}{11}+\frac{5 \sqrt{78}}{12}+\frac{3 \sqrt{110}}{10}+\frac{\sqrt{170}}{15}+\frac{\sqrt{210}}{15}-\frac{4 \sqrt{357}}{21}-\frac{2 \sqrt{374}}{11}+\frac{6 \sqrt{897}}{13}-\frac{2 \sqrt{2730}}{13}-4$.

The fifth geometric arithmetic index $G A_{5}$ of $C G(m, n)$ is computed in the following theorem.

Theorem 2.6. Consider the graph of carbon graphite $C G(m, n)$ with $m, n>2$ and $t \geq 3$, then its fifth geometric arithmetic index is equal to

$G A_{5}(G)=m\left(\frac{16 \sqrt{5}}{9}-\frac{48 \sqrt{13}}{29}+\frac{8 \sqrt{30}}{11}-\frac{88 \sqrt{39}}{25}+\frac{8 \sqrt{130}}{23}+15\right)-n\left(\frac{24 \sqrt{3}}{7}+\frac{16 \sqrt{6}}{5}-\frac{16 \sqrt{11}}{27}+\frac{32 \sqrt{13}}{29}-\frac{16 \sqrt{22}}{19}-\right.$ $\left.\frac{8 \sqrt{26}}{21}-\frac{8 \sqrt{33}}{23}+\frac{24 \sqrt{39}}{25}-14\right)+m n\left(\frac{16 \sqrt{13}}{29}+\frac{24 \sqrt{39}}{25}-9\right)+t\left(\frac{16(n-1) \sqrt{3}}{7}+\frac{8(n-2) \sqrt{6}}{5}+\frac{16(m-1) \sqrt{13}}{29}+\frac{24(m-2) \sqrt{39}}{25}+\right.$ $\left.\frac{16 \sqrt{5}}{9}+\frac{8 \sqrt{14}}{15}+\frac{16 \sqrt{21}}{19}+\frac{4 \sqrt{70}}{17}+\frac{12 \sqrt{130}}{23}+4 m n-5 m-6 n+7\right)+\frac{8 \sqrt{3}}{7}-\frac{64 \sqrt{5}}{9}+\frac{32 \sqrt{6}}{5}+\frac{3 \sqrt{7}}{4}+\frac{612 \sqrt{10}}{247}-$ $\frac{16 \sqrt{11}}{27}+\frac{878 \sqrt{13}}{319}-\frac{16 \sqrt{14}}{15}+\frac{\sqrt{15}}{2}-\frac{32 \sqrt{21}}{19}-\frac{32 \sqrt{22}}{19}-\frac{8 \sqrt{26}}{21}-\frac{8 \sqrt{30}}{11}-\frac{8 \sqrt{33}}{23}+\frac{144 \sqrt{39}}{25}+\frac{4 \sqrt{77}}{9}-\frac{40 \sqrt{130}}{23}-14$.

Proof. Let $G \cong C G(m, n)$ be a graph of carbon graphite. The above result can be proved by using Table 2 in the following computation. The fifth geometric arithmetic index is computed as below:

$$
G A_{5}(G)=\sum_{u v \in E(G)} \frac{2 \sqrt{S_{u} S_{v}}}{S_{u}+S_{v}}
$$

$G A_{5}(G)=(2) \frac{2 \sqrt{5 \times 5}}{5+5}+(2) \frac{2 \sqrt{5 \times 6}}{5+6}+(6) \frac{2 \sqrt{5 \times 8}}{5+8}+4(n-2) \frac{2 \sqrt{6 \times 8}}{6+8}+(2) \frac{2 \sqrt{6 \times 10}}{6+10}+(4) \frac{2 \sqrt{7 \times 7}}{7+7}+2(t-2) \frac{2 \sqrt{7 \times 8}}{7+8}+$ (2) $\frac{2 \sqrt{7 \times 9}}{7+9}+(2 t) \frac{2 \sqrt{7 \times 10}}{7+10}+(4) \frac{2 \sqrt{7 \times 11}}{7+11}+4(t-2) \sqrt{\frac{7+12-2}{7 \times 12}}+(2) \frac{2 \sqrt{8 \times 8}}{8+8}+4(m+t-4) \frac{2 \sqrt{8 \times 10}}{8+10}+4(n-2) \frac{2 \sqrt{8 \times 11}}{8+11}+$ $4(n t-2 n-2 t+4) \frac{2 \sqrt{8 \times 12}}{8+12}+2(n-1) \frac{2 \sqrt{8 \times 13}}{8+13}+(2) \frac{2 \sqrt{9 \times 10}}{9+10}+(2) \frac{2 \sqrt{9 \times 13}}{9+13}+(4 m-6) \frac{2 \sqrt{10 \times 10}}{10+10}+(4 m-6) \frac{2 \sqrt{10 \times 12}}{10+12}+$ $(4 m+6 t-20) \frac{2 \sqrt{10 \times 13}}{10+13}+2(n-1) \frac{2 \sqrt{11 \times 12}}{11+12}+2(n-1) \frac{2 \sqrt{11 \times 16}}{11+16}+(6 m n+6 m t-22 m-6 n-12 t+36) \frac{2 \sqrt{12 \times 13}}{12+13}+$ $(4 n t-10 n-4 t+10) \frac{2 \sqrt{12 \times 16}}{12+16}+(2 m n+2 m t-6 m-4 n-2 t+8) \frac{2 \sqrt{13 \times 16}}{13+16}+(4 m n t-5 m t-6 n t-9 m n+$ $11 m+14 n+7 t-16) \frac{2 \sqrt{16 \times 16}}{16+16}$

After an easy calculation, we get

$$
G A_{5}(G)=m\left(\frac{16 \sqrt{5}}{9}-\frac{48 \sqrt{13}}{29}+\frac{8 \sqrt{30}}{11}-\frac{88 \sqrt{39}}{25}+\frac{8 \sqrt{130}}{23}+15\right)-n\left(\frac{24 \sqrt{3}}{7}+\frac{16 \sqrt{6}}{5}-\frac{16 \sqrt{11}}{27}+\frac{32 \sqrt{13}}{29}-\frac{16 \sqrt{22}}{19}-\right.
$$


$\left.\frac{8 \sqrt{26}}{21}-\frac{8 \sqrt{33}}{23}+\frac{24 \sqrt{39}}{25}-14\right)+m n\left(\frac{16 \sqrt{13}}{29}+\frac{24 \sqrt{39}}{25}-9\right)+t\left(\frac{16(n-1) \sqrt{3}}{7}+\frac{8(n-2) \sqrt{6}}{5}+\frac{16(m-1) \sqrt{13}}{29}+\frac{24(m-2) \sqrt{39}}{25}+\right.$
$\left.\frac{16 \sqrt{5}}{9}+\frac{8 \sqrt{14}}{15}+\frac{16 \sqrt{21}}{19}+\frac{4 \sqrt{70}}{17}+\frac{12 \sqrt{130}}{23}+4 m n-5 m-6 n+7\right)+\frac{8 \sqrt{3}}{7}-\frac{64 \sqrt{5}}{9}+\frac{32 \sqrt{6}}{5}+\frac{3 \sqrt{7}}{4}+\frac{612 \sqrt{10}}{247}-$
$\frac{16 \sqrt{11}}{27}+\frac{878 \sqrt{13}}{319}-\frac{16 \sqrt{14}}{15}+\frac{\sqrt{15}}{2}-\frac{32 \sqrt{21}}{19}-\frac{32 \sqrt{22}}{19}-\frac{8 \sqrt{26}}{21}-\frac{8 \sqrt{30}}{11}-\frac{8 \sqrt{33}}{23}+\frac{144 \sqrt{39}}{25}+\frac{4 \sqrt{77}}{9}-\frac{40 \sqrt{130}}{23}-14$.

\subsection{Crystal Structure Cubic Carbon}

The structure of crystal cubic carbon consist of cubes. The molecular graph of crystal cubic carbon $C C C(n)$ for first level is depicted in Fig. 3. For second level, the cube is constructed at every end vertex of first level. The second level of $C C C(n)$ is depicted in Fig. 4. Similarly, continuing this procedure to get the next level and so on. The cardinality of vertices and edges in $C C C(n)$ are given below respectively.

$$
\begin{aligned}
& |V(C C C(n))|=2\left(24 \sum_{r=3}^{n}\left(2^{3}-1\right)^{r-3}+31\left(2^{3}-1\right)^{n-2}+2 \sum_{r=0}^{n-2}\left(2^{3}-1\right)^{r}+3\right) . \\
& |E(C C C(n))|=4\left(24 \sum_{r=3}^{n}\left(2^{3}-1\right)^{r-3}+24\left(2^{3}-1\right)^{n-2}+2 \sum_{r=0}^{n-2}\left(2^{3}-1\right)^{r}+3\right) .
\end{aligned}
$$

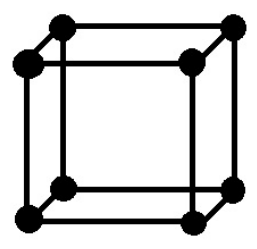

Fig. 3: Crystal Structure Cubic Carbon $C C C(1)$.

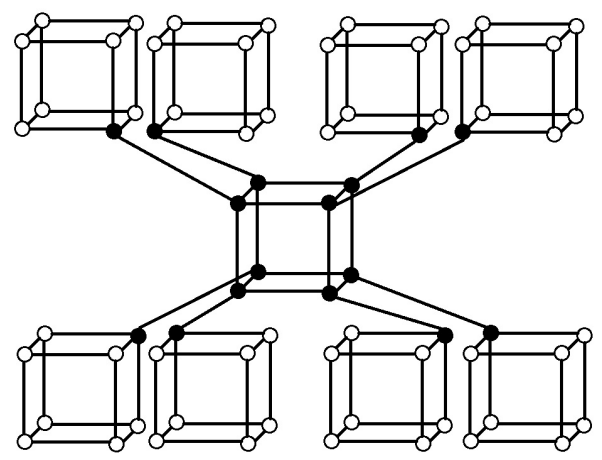

Fig. 4: Crystal Structure Cubic Carbon $C C C(2)$.

In $C C C(n)$, the 3 degree vertices are $\operatorname{deg}(3)=8\left(2^{3}-1\right)^{n-1}$ and the 4 degree vertices are $\operatorname{deg}(4)=$ $2\left(24 \sum_{r=3}^{n}\left(2^{3}-1\right)^{r-3}+3\left(\left(2^{3}-1\right)^{n-2}+1\right)+2 \sum_{r=0}^{n-2}\left(2^{3}-1\right)^{r}+3\right)$. The $r$ is the levels of crystal cubic 
carbon, where $3 \leq r \leq n$. The edge set of $C C C(n)$ is divided into three partitions based on the degree of end vertices. The first edge partition contains $72\left(2^{3}-1\right)^{n-2}$ edges $u v$, where $\operatorname{deg}(u)=\operatorname{deg}(v)=3$. The second edge partition contains $24\left(2^{3}-1\right)^{n-2}$ edges $u v$, where $\operatorname{deg}(u)=3$ and $\operatorname{deg}(v)=4$. The third edge partition contains $12\left(1+\sum_{r=3}^{n} 2^{3}\left(2^{3}-1\right)^{r-3}\right)+8 \sum_{r=0}^{n-2}\left(2^{3}-1\right)^{r}$ edges $u v$, where $\operatorname{deg}(u)=\operatorname{deg}(v)=4$. The Table 3 shows such an edge partition of $C C C(n)$ with $n>2$.

\begin{tabular}{|c|c|}
\hline$\left(d_{u}, d_{v}\right)$ & frequency \\
\hline$(3,3)$ & $72\left(2^{3}-1\right)^{n-2}$ \\
\hline$(3,4)$ & $24\left(2^{3}-1\right)^{n-2}$ \\
\hline$(4,4)$ & $12\left(1+\sum_{r=3}^{n} 2^{3}\left(2^{3}-1\right)^{r-3}\right)+8 \sum_{r=0}^{n-2}\left(2^{3}-1\right)^{r}$ \\
\hline
\end{tabular}

Table 3: Edge partition of $C C C(n)$ based on degrees of end vertices of each edge.

In the coming theorem, we compute atom bond connectivity index of $C C C(n)$.

Theorem 2.1. Consider the graph of crystal cubic carbon $C C C(n)$ with $n>2$, then its atom bond connectivity index is equal to

$$
A B C(G)=2 \sqrt{6}\left(12 \sum_{r=3}^{n}\left(2^{3}-1\right)^{r-3}+\sum_{r=0}^{n-2}\left(2^{3}-1\right)^{r}\right)+4\left(2^{3}-1\right)^{n-2}(12+\sqrt{15})+3 \sqrt{6}
$$

Proof. Let $G \cong C C C(n)$ be a graph of crystal cubic carbon. The edge partition of $C C C(n)$ based on degrees of end vertices of each edge with their frequencies is given in Table 3 . The atom bond connectivity index can be calculated by using Table 3. in the following equation.

$$
A B C(G)=\sum_{u v \in E(G)} \sqrt{\frac{d_{u}+d_{v}-2}{d_{u} d_{v}}}
$$

$A B C(G)=\left(72\left(2^{3}-1\right)^{n-2}\right) \sqrt{\frac{3+3-2}{3 \times 3}}+\left(24\left(2^{3}-1\right)^{n-2}\right) \sqrt{\frac{3+4-2}{3 \times 4}}+\left(12\left(1+\sum_{r=3}^{n} 2^{3}\left(2^{3}-1\right)^{r-3}\right)+8 \sum_{r=0}^{n-2}\left(2^{3}-\right.\right.$ $\left.1)^{r}\right) \sqrt{\frac{4+4-2}{4 \times 4}}$

After an easy calculation, we get

$A B C(G)=2 \sqrt{6}\left(12 \sum_{r=3}^{n}\left(2^{3}-1\right)^{r-3}+\sum_{r=0}^{n-2}\left(2^{3}-1\right)^{r}\right)+4\left(2^{3}-1\right)^{n-2}(12+\sqrt{15})+3 \sqrt{6}$

In the next theorem, we compute Randić index $R_{\alpha}(G)$ of crystal cubic carbon $C C C(n)$. 
Theorem 2.2. Consider the graph of crystal cubic carbon $C C C(n)$ with $n>2$, then its general Randic index is equal to

$$
R_{\alpha} C C C(n)= \begin{cases}936\left(2^{3}-1\right)^{n-2}+128\left(12 \sum_{r=3}^{n}\right. \\ \left.\left(2^{3}-1\right)^{r-3}+\sum_{r=0}^{n-2}\left(2^{3}-1\right)^{r}\right)+192, & \text { if } \alpha=1, \\ 10\left(2^{3}-1\right)^{n-2}+6 \sum_{r=3}^{n}\left(2^{3}-1\right)^{r-3} & \\ +\frac{1}{2} \sum_{r=0}^{n-2}\left(2^{3}-1\right)^{r}+\frac{3}{4}, & \text { if } \alpha=-1, \\ \left(2^{3}-1\right)^{n-2}(216+48 \sqrt{3})+32\left(12 \sum_{r=3}^{n}\right. & \\ \left.\left(2^{3}-1\right)^{r-3}+\sum_{r=0}^{n-2}\left(2^{3}-1\right)^{r}\right)+48, & \text { if } \alpha=\frac{1}{2}, \\ \left(2^{3}-1\right)^{n-2}(24+4 \sqrt{3})+2\left(12 \sum_{r=3}^{n}\right. & \\ \left.\left(2^{3}-1\right)^{r-3}+\sum_{r=0}^{n-2}\left(2^{3}-1\right)^{r}\right)+3, & \text { if } \alpha=-\frac{1}{2} .\end{cases}
$$

Proof. Let $G \cong C C C(n)$ be a graph of crystal cubic carbon. The above result can be proved by using Table 3 in the following computation. The general Randić index for $\alpha=1$.

$$
\begin{gathered}
R_{1}(G)=\sum_{u v \in E(G)}\left(d_{u} \times d_{v}\right) \\
R_{1}(G)=\left(72\left(2^{3}-1\right)^{n-2}\right)(3 \times 3)+\left(24\left(2^{3}-1\right)^{n-2}\right)(3 \times 4)+\left(12\left(1+\sum_{r=3}^{n}\left(2^{3}-1\right)^{r-3}\right)+8 \sum_{r=0}^{n-2}\left(2^{3}-\right.\right. \\
\left.\left.1)^{r}\right)\right)(4 \times 4) \\
R_{1}(G)=936\left(2^{3}-1\right)^{n-2}+128\left(12 \sum_{r=3}^{n}\left(2^{3}-1\right)^{r-3}+\sum_{r=0}^{n-2}\left(2^{3}-1\right)^{r}\right)+192 .
\end{gathered}
$$

For $\alpha=-1$, the formula of Randić index takes the following form.

$$
\begin{gathered}
R_{-1}(G)=\sum_{u v \in E(G)} \frac{1}{\left(d_{u} \times d_{v}\right)} \\
R_{-1}(G)=\left(72\left(2^{3}-1\right)^{n-2}\right) \frac{1}{(3 \times 3)}+\left(24\left(2^{3}-1\right)^{n-2}\right) \frac{1}{(3 \times 4)}+\left(12\left(1+\sum_{r=3}^{n}\left(2^{3}-1\right)^{r-3}\right)+8 \sum_{r=0}^{n-2}\left(2^{3}-1\right)^{r}\right) \frac{1}{(4 \times 4)} \\
R_{-1}(G)=10\left(2^{3}-1\right)^{n-2}+6 \sum_{r=3}^{n}\left(2^{3}-1\right)^{r-3}+\frac{1}{2} \sum_{r=0}^{n-2}\left(2^{3}-1\right)^{r}+\frac{3}{4}
\end{gathered}
$$

For $\alpha=\frac{1}{2}$, the formula of Randić index takes the following form.

$$
R_{\frac{1}{2}}(G)=\sum_{u v \in E(G)} \sqrt{\left(d_{u} \times d_{v}\right)}
$$


$R_{\frac{1}{2}}(G)=\left(72\left(2^{3}-1\right)^{n-2}\right) \sqrt{(3 \times 3)}+\left(24\left(2^{3}-1\right)^{n-2}\right) \sqrt{(3 \times 4)}+\left(12\left(1+\sum_{r=3}^{n}\left(2^{3}-1\right)^{r-3}\right)+8 \sum_{r=0}^{n-2}\left(2^{3}-\right.\right.$ $\left.1)^{r}\right) \sqrt{(4 \times 4)}$

$R_{\frac{1}{2}}(G)=\left(2^{3}-1\right)^{n-2}(216+48 \sqrt{3})+32\left(12 \sum_{r=3}^{n}\left(2^{3}-1\right)^{r-3}+\sum_{r=0}^{n-2}\left(2^{3}-1\right)^{r}\right)+48$.

For $\alpha=-\frac{1}{2}$, the formula of Randić index takes the following form.

$$
R_{-\frac{1}{2}}(G)=\sum_{u v \in E(G)} \frac{1}{\sqrt{\left(d_{u} \times d_{v}\right)}}
$$

$R_{-\frac{1}{2}}(G)=\left(72\left(2^{3}-1\right)^{n-2}\right) \frac{1}{\sqrt{(3 \times 3)}}+\left(24\left(2^{3}-1\right)^{n-2}\right) \frac{1}{\sqrt{(3 \times 4)}}+\left(12\left(1+\sum_{r=3}^{n}\left(2^{3}-1\right)^{r-3}\right)+8 \sum_{r=0}^{n-2}\left(2^{3}-\right.\right.$ 1) $\frac{1}{\sqrt{(4 \times 4)}}$

$R_{-\frac{1}{2}}(G)=\left(2^{3}-1\right)^{n-2}(24+4 \sqrt{3})+2\left(12 \sum_{r=3}^{n}\left(2^{3}-1\right)^{r-3}+\sum_{r=0}^{n-2}\left(2^{3}-1\right)^{r}\right)+3$

A close formula of geometric arithmetic index $G A$ of $C C C(n)$ is computed in the following theorem.

Theorem 2.3. Consider the graph of crystal cubic carbon $C C C(n)$, for $n>2$, then its geometric arithmetic index is equal to

$G A(G)=8\left(12 \sum_{r=3}^{n}\left(2^{3}-1\right)^{r-3}+\sum_{r=0}^{n-2}\left(2^{3}-1\right)^{r}\right)+\left(2^{3}-1\right)^{n-2}\left(72+\frac{96 \sqrt{3}}{7}\right)+12$.

Proof. Let $G \cong C C C(n)$ be a graph of crystal cubic carbon. The above result can be proved by using Table 3 in the following computation. The geometric arithmetic index is computed as below:

$$
G A(G)=\sum_{u v \in E(G)} \frac{2 \sqrt{d_{u} d_{v}}}{d_{u}+d_{v}}
$$

$G A(G)=\left(72\left(2^{3}-1\right)^{n-2}\right) \frac{2 \sqrt{3 \times 3}}{3+3}+\left(24\left(2^{3}-1\right)^{n-2}\right) \frac{2 \sqrt{3 \times 4}}{3+4}+\left(12\left(1+\sum_{r=3}^{n}\left(2^{3}-1\right)^{r-3}\right)+8 \sum_{r=0}^{n-2}\left(2^{3}-\right.\right.$ 1) $) \frac{2 \sqrt{4 \times 4}}{4+4}$

After an easy calculation, we get

$G A(G)=8\left(12 \sum_{r=3}^{n}\left(2^{3}-1\right)^{r-3}+\sum_{r=0}^{n-2}\left(2^{3}-1\right)^{r}\right)+\left(2^{3}-1\right)^{n-2}\left(72+\frac{96 \sqrt{3}}{7}\right)+12$.

Theorem 2.4. Consider the graph of crystal cubic carbon $C C C(n)$ with $n>2$, then its first and second Zagreb index is equal to

$$
M_{1}(G)=600\left(2^{3}-1\right)^{n-2}+64\left(12 \sum_{r=3}^{n}\left(2^{3}-1\right)^{r-3}+\sum_{r=0}^{n-2}\left(2^{3}-1\right)^{r}\right)+96
$$




$$
M_{2}(G)=936\left(2^{3}-1\right)^{n-2}+128\left(12 \sum_{r=3}^{n}\left(2^{3}-1\right)^{r-3}+\sum_{r=0}^{n-2}\left(2^{3}-1\right)^{r}\right)+192 .
$$

Proof. Let $G \cong C C C(n)$ be a graph of crystal cubic carbon. The above result can be proved by using Table 3 in the following computation. The first Zagreb index is computed as below:

$$
\begin{aligned}
& M_{1}(G)=\sum_{u v \in E(G)}\left(d_{u}+d_{v}\right) \\
& M_{1}(G)=\left(72\left(2^{3}-1\right)^{n-2}\right)(3+3)+\left(24\left(2^{3}-1\right)^{n-2}\right)(3+4)+\left(12\left(1+\sum_{r=3}^{n}\left(2^{3}-1\right)^{r-3}\right)+8 \sum_{r=0}^{n-2}\left(2^{3}-1\right)^{r}\right)(4+4) . \\
& M_{1}(G)=600\left(2^{3}-1\right)^{n-2}+64\left(12 \sum_{r=3}^{n}\left(2^{3}-1\right)^{r-3}+\sum_{r=0}^{n-2}\left(2^{3}-1\right)^{r}\right)+96 . \text { The second Zagreb index is } \\
& \text { computed as below: } \\
& \qquad M_{2}(G)=\sum_{u v \in E(G)}\left(d_{u} d_{v}\right) . \\
& M_{2}(G)=\left(72\left(2^{3}-1\right)^{n-2}\right)(3 \times 3)+\left(24\left(2^{3}-1\right)^{n-2}\right)(3 \times 4)+\left(12\left(1+\sum_{r=3}^{n}\left(2^{3}-1\right)^{r-3}\right)+8 \sum_{r=0}^{n-2}\left(2^{3}-1\right)^{r}\right)(4 \times 4) . \\
& M_{2}(G)=936\left(2^{3}-1\right)^{n-2}+128\left(12 \sum_{r=3}^{n}\left(2^{3}-1\right)^{r-3}+\sum_{r=0}^{n-2}\left(2^{3}-1\right)^{r}\right)+192 .
\end{aligned}
$$

The Table 4 shows the edge partition based on the degree sum of end vertices of each edge. We compute fourth atom bond connectivity index and fifth geometric arithmetic index by using Table 4 .

\begin{tabular}{|c|c|}
\hline$\left(S_{u}, S_{v}\right)$ & Number of edges \\
\hline$(9,9)$ & $24\left(2^{3}-1\right)^{n-2}$ \\
\hline$(9,10)$ & $48\left(2^{3}-1\right)^{n-2}$ \\
\hline$(10,13)$ & $24\left(2^{3}-1\right)^{n-2}$ \\
\hline$(13,16)$ & $2^{3}\left(2^{3}-1\right)^{n-2}$ \\
\hline$(16,16)$ & $4\left(24 \sum_{r=3}^{n}\left(2^{3}-1\right)^{r-3}+2 \sum_{r=0}^{n-2}\left(2^{3}-1\right)^{r}-2\left(2^{3}-1\right)^{n-2}+3\right)$ \\
\hline
\end{tabular}

Table 4: Edge partition of $C C C(n)$ based on degree sum of end vertices of each edge.

A close formula of fourth version of atom bond connectivity index $A B C_{4}$ of $C C C(n)$ is computed in the following theorem.

Theorem 2.5. Consider the graph of crystal cubic carbon $C C C(n)$ with $n>2$, then its fourth atom bond connectivity index is equal to

$$
\begin{aligned}
& A B C_{4}(G)=\frac{\sqrt{30}}{2}\left(12 \sum_{r=3}^{n}\left(2^{3}-1\right)^{r-3}+\sum_{r=0}^{n-2}\left(2^{3}-1\right)^{r}\right)+\left(2^{3}-1\right)^{n-2}\left(\frac{32}{3}-\frac{\sqrt{30}}{2}+\frac{6 \sqrt{39}}{13}+\frac{8 \sqrt{170}}{5}+\right. \\
& \left.\frac{12 \sqrt{2730}}{65}\right)+\frac{3 \sqrt{30}}{4} .
\end{aligned}
$$


Proof. Let $G \cong C C C(n)$ be a graph of crystal cubic carbon. The above result can be proved by using Table 4 in the following computation. The fourth atom bond connectivity index is computed as below:

$$
A B C_{4}(G)=\sum_{u v \in E(G)} \sqrt{\frac{S_{u}+S_{v}-2}{S_{u} S_{v}}}
$$

$A B C_{4}(G)=\left(24\left(2^{3}-1\right)^{n-2}\right) \sqrt{\frac{9+9-2}{9 \times 9}}+\left(48\left(2^{3}-1\right)^{n-2}\right) \sqrt{\frac{9+10-2}{9 \times 10}}+\left(24\left(2^{3}-1\right)^{n-2}\right) \sqrt{\frac{10+13-2}{10 \times 13}}+\left(2^{3}\left(2^{3}-\right.\right.$
$\left.1)^{n-2}\right) \sqrt{\frac{13+16-2}{13 \times 16}}+4\left(24 \sum_{r=3}^{n}\left(2^{3}-1\right)^{r-3}+2 \sum_{r=0}^{n-2}\left(2^{3}-1\right)^{r}-2\left(2^{3}-1\right)^{n-2}+3\right) \sqrt{\frac{16+16-2}{16 \times 16}}$

After an easy calculation, we get

$A B C_{4}(G)=\frac{\sqrt{30}}{2}\left(12 \sum_{r=3}^{n}\left(2^{3}-1\right)^{r-3}+\sum_{r=0}^{n-2}\left(2^{3}-1\right)^{r}\right)+\left(2^{3}-1\right)^{n-2}\left(\frac{32}{3}-\frac{\sqrt{30}}{2}+\frac{6 \sqrt{39}}{13}+\frac{8 \sqrt{170}}{5}+\right.$
$\left.\frac{12 \sqrt{2730}}{65}\right)+\frac{3 \sqrt{30}}{4}$ The fifth version of geometric arithmetic index $G A_{5}$ of $C C C(n)$ is computed in the following theorem.

Theorem 2.6. Consider the graph of crystal cubic carbon $C C C(n)$ with $n>2$, then its fifth geometric arithmetic index is equal to

$G A_{5}(G)=8\left(12 \sum_{r=3}^{n}\left(2^{3}-1\right)^{r-3}+\sum_{r=0}^{n-2}\left(2^{3}-1\right)^{r}\right)+\left(2^{3}-1\right)^{n-2}\left(\frac{288 \sqrt{10}}{19}+\frac{64 \sqrt{13}}{29}+\frac{48 \sqrt{130}}{23}+16\right)+12$.

Proof. Let $G \cong C C C(n)$ be a graph of crystal cubic carbon. The above result can be proved by using

Table 4 in the following computation. The fifth geometric arithmetic index is computed as below:

$$
G A_{5}(G)=\sum_{u v \in E(G)} \frac{2 \sqrt{S_{u} S_{v}}}{S_{u}+S_{v}}
$$

$G A_{5}(G)=\left(24\left(2^{3}-1\right)^{n-2}\right) \frac{2 \sqrt{9 \times 9}}{9+9}+\left(48\left(2^{3}-1\right)^{n-2}\right) \frac{2 \sqrt{9 \times 10}}{9+10}+\left(24\left(2^{3}-1\right)^{n-2}\right) \frac{2 \sqrt{10 \times 13}}{10+13}+\left(2^{3}\left(2^{3}-1\right)^{n-2}\right) \frac{2 \sqrt{13 \times 16}}{13+16}+$ $4\left(24 \sum_{r=3}^{n}\left(2^{3}-1\right)^{r-3}+2 \sum_{r=0}^{n-2}\left(2^{3}-1\right)^{r}-2\left(2^{3}-1\right)^{n-2}+3\right) \frac{2 \sqrt{16 \times 16}}{16+16}$

After an easy calculation, we get

$G A_{5}(G)=8\left(12 \sum_{r=3}^{n}\left(2^{3}-1\right)^{r-3}+\sum_{r=0}^{n-2}\left(2^{3}-1\right)^{r}\right)+\left(2^{3}-1\right)^{n-2}\left(\frac{288 \sqrt{10}}{19}+\frac{64 \sqrt{13}}{29}+\frac{48 \sqrt{130}}{23}+16\right)+12$.

The graphical representation of topological indices of carbon graphite $C G(m, n) t$-levels is depicted for certain values of $(m, n)$ and fixing value of $t=10$. By increasing the value of $t$ the value of indices increases. The comparison of indices as computed above mainly first and second Zagreb index, atom bond connectivity $A B C$ index, geometric arithmetic $G A$ index, fourth atom bond connectivity $A B C_{4}$ index, fifth geometric arithmetic $G A_{5}$ index and general Randić index for $\alpha=\left\{1,-1, \frac{1}{2}, \frac{-1}{2}\right\}$ are depicted in the Fig. 5-6. 


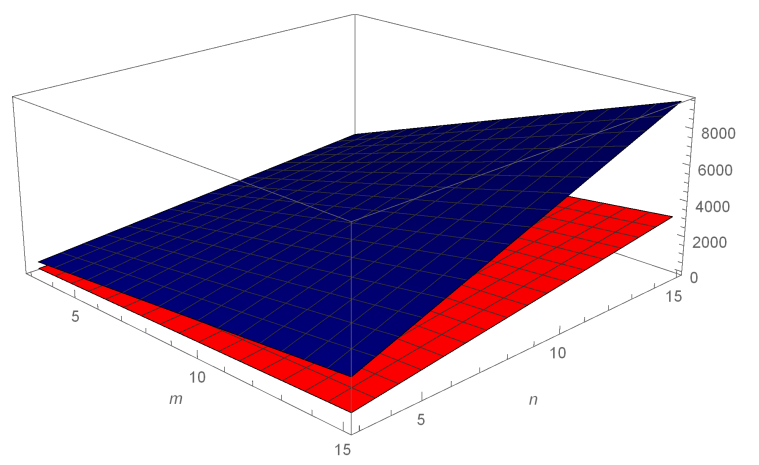

Fig. 5: The comparison of $A B C_{4}$ and $G A_{5}$ index of carbon graphite graph $C G(m, n)$ for $t$-levels, Blue $\rightarrow$ $A B C_{4}$, Red $\rightarrow G A_{5}$.

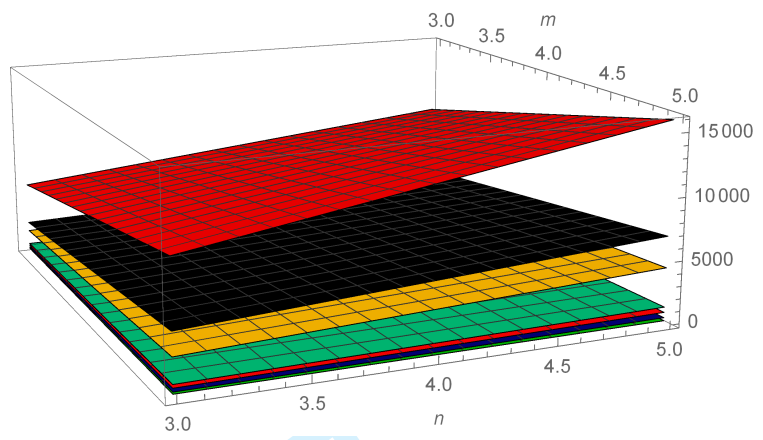

Fig. 6: The comparison of general Randić index for $\alpha=\left\{1,-1, \frac{1}{2},-\frac{1}{2}\right\}, A B C$ index, $G A$ index and first Zagreb $M_{1}$ index of carbon graphite graph $C G(m, n)$ for $t$-levels, Red $\rightarrow R_{1}$, Blue $\rightarrow R_{-\frac{1}{2}}$, Green $\rightarrow$ $R_{-1}$, Black $\rightarrow M_{1}$, Yellow $\rightarrow R_{\frac{1}{2}}$, Cyan $\rightarrow G A$, Magenta $\rightarrow A B C$.

The graphical representation of topological indices of crystal structure cubic carbon $C C C(n)$ is depicted in Fig. 7-8, for certain values of $(n)$. By increasing the value of $n$, indices of graph increases and shows different behavior.

\section{Conclusion and general remarks}

We have studied and computed additive degree based topological indices mainly first and second Zagreb index, atom bond connectivity $A B C$ index, geometric arithmetic $G A$ index, fourth atom bond connectivity $A B C_{4}$ index, fifth geometric arithmetic $G A_{5}$ index and general Randić index of carbon graphite $C G(m, n) t$-levels and crystal structure cubic carbon $C C C(n)$ chemical graphs. Also, close results of additive topological indices for $C G(m, n)$ and $C C C(n)$ are computed.

\subsection{Acknowledgment}

The authors would like to thank the referees for their constructive suggestions and useful comments which resulted in an improved version of this paper. This research is supported by the Start Up Research Grant 2016 of United Arab Emirates University, Al Ain, United Arab Emirates via Grant No. G00002233. 


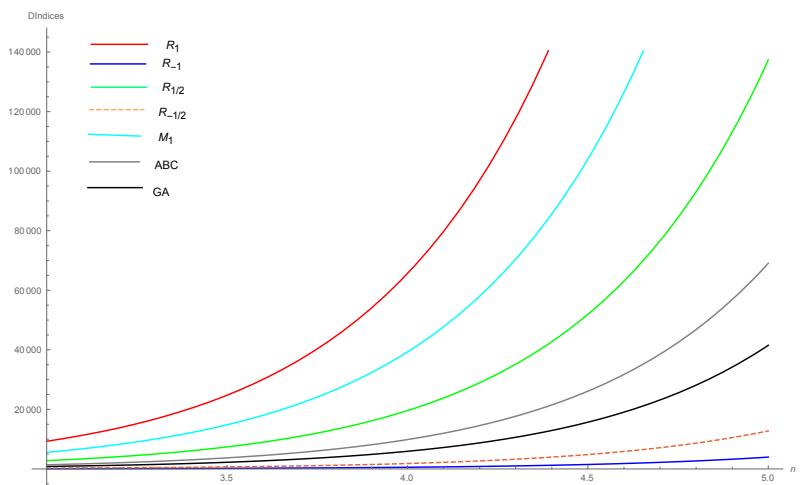

Fig. 7: The comparison of general Randić index for $\alpha=\left\{1,-1, \frac{1}{2},-\frac{1}{2}\right\}$, first Zagreb $M_{1}, A B C$ and $G A$ index of crystal structure cubic carbon $C C C(n)$.

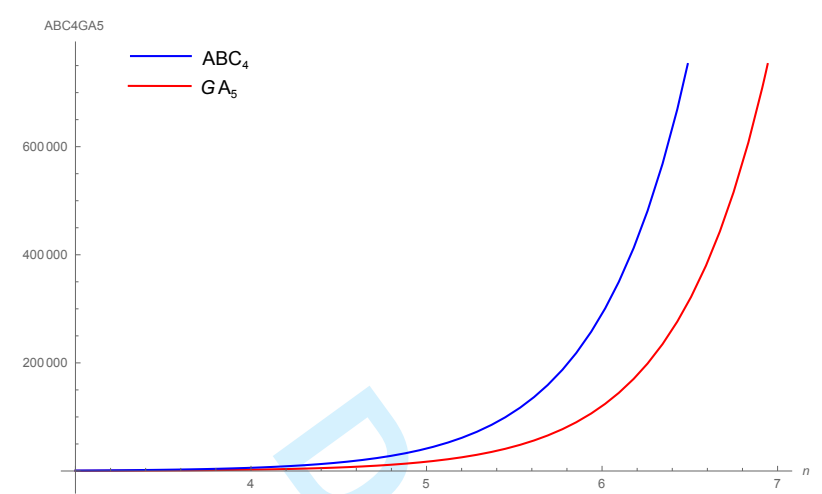

Fig. 8: The comparison of $A B C_{4}$ and $G A_{5}$ index of crystal structure cubic carbon $C C C(n)$.

\section{References}

1. Ali, A.; Bhatti, A. A.; Raza, Z.; Quantum Matter 2016, 5, 534.

2. Ali, A.; Bhatti, A. A.; Raza, Z.; Bull. Iranian Math. Soc., 2016, 42, 417.

3. Ali, A.; Raza, Z.; Bhatti, A. A.; Kuwait Journal of Science 2016, 43, 123.

4. Ali, A.; Raza, Z.; Bhatti, A. A.; Applied Mathematics and Computation 2016, 287, 28.

5. Ali, A.; Raza, Z.; Bhatti, A. A.; Canadian Journal of Chemistry 2016, 94, 870.

6. Ali, A.; Raza, Z.; Bhatti, A. A.; Optoelectronics Adv. Materials-Rapid Commun., 2016, 10, 108.

7. Amic, D.; Beslo, D.; Lucic, B.; Nikolic, S.; Trinajstić, N.; J. Chem. Inf. Comput. Sci. 1998, 38, 819.

8. Bollobás, B.; Erdös, P.; Ars Combinatoria 1998, 50, 225.

9. Baig, A. Q.; Imran, M.; Ali, H.; Canadian Journal of chemistry 2015, 93, 730.

10. Caporossi, G.; Gutman, I.; Hansen, P.; Pavlovíc, L.; Comput. Bio. Chem. 2003, 27, 85.

11. Estrada, E.; Torres, L.; Rodríguez, L.; Gutman, I.; Indian J. Chem. 1998, 37A, 849.

12. Farahani, M. R.; Acta Chimica Slovenica 2013,60, 429.

13. Gao, W.; Wang, W.; Farahani, M. R.; Journal of Chemistry 2016, 10, DOI: 10.1155/2016/3216327.

14. Ghorbani, A.; Hosseinzadeh, M. A.; Optoelectronics and Advanced Materials-Rapid Communications 2010, 4, 1419 .

15. Graovac, A.; Ghorbani, M.; Hosseinzadeh, M. A.; J. Math. Nanosci. 2011, 1, 33.

16. Gutman, I.; Das, K. C.; MATCH Commun. Math. Comput. Chem. 2004, 50, 83. 
17. Gutman, I.; Trinajstć, N.; Chemical Physics Letters 1972, 17, 535.

18. Hayat, S.; Malik, M. A.; Imran, M.; Romanian journal of Information science and technology 2015, $18,144$.

19. Hayat, S.; Imran, M.; J. Comput. Theor. Nanosci. 2015, 12, 533.

20. Hu, Y.; Li, X.; Shi, Y.; Xu, T.; Gutman, I.; MATCH Commun. Math. Comput. Chem. 2005, 54, 425.

21. Li, X.; Gutman, I.; Mathematical aspects of Randić type molecular structure descriptors, mathematical chemistry monographs No. 1, Kragujevac, 2006.

22. Pierson, H. O.; Handbook of Carbon, Graphite, Diamonds and Fullerenes: Processing, Properties and Applications, NOYES Publications, Park Ridge, New Jersey, U.S.A, 2012.

23. Rajan, B.; William, A.; Grigorious, C.; Stephen, S.; J. Comp. and Math. Sci. 2012, 3, 530.

24. Randić, M.; J. Amer. Chem. Soc. 1975,97, 6609.

25. Raza, Z.; Journal of Computational and Theoretical Nanoscience 2016, 13, 1.

26. Raza, Z.; Bhatti, A. A.; Ali, A.; Miskolc Mathematical Notes 2016, 17, 561.

27. Rouvray, D. H.; Journal of computational chemistry 1987, 8, 470.

28. Soleimani, N.; Nikmehr, M. J.; Tavallaee, H. A.; J. Natn. Sci. Foundation, Sri Lanka 2015, 43, 127.

29. Vajtai, R.; Springer Handbook of Nanomaterials, Springer Science \& Business Media, 2013.

30. Vukičević, D.; Furtula, B.; J. Math. Chem., 2009, 46, 1369. 


\section{Figure Captions}

Fig.1: Carbon graphite $C G(m, n) t$-levels.

Fig.2: Carbon graphite $C G(3,4)$ and $t=3$.

Fig.3: Crystal Structure Cubic Carbon $C C C(1)$.

Fig.4: Crystal Structure Cubic Carbon $C C C(2)$.

Fig.5: The comparison of $A B C_{4}$ and $G A_{5}$ index of carbon graphite graph $C G(m, n)$ for $t$-levels. Blue $\rightarrow$ $A B C_{4}$, Red $\rightarrow G A_{5}$.

Fig.6: The comparison of general Randić index for $\alpha=\left\{1,-1, \frac{1}{2},-\frac{1}{2}\right\}, A B C$ index, $G A$ index and first Zagreb $M_{1}$ index of carbon graphite graph $C G(m, n)$ for $t$-levels, Red $\rightarrow R_{1}$, Blue $\rightarrow R_{-\frac{1}{2}}$, Green $\rightarrow$ $R_{-1}$, Black $\rightarrow M_{1}$, Yellow $\rightarrow R_{\frac{1}{2}}$, Cyan $\rightarrow G A$, Magenta $\rightarrow A B C$.

Fig.7: The comparison of general Randić index for $\alpha=\left\{1,-1, \frac{1}{2},-\frac{1}{2}\right\}$, first Zagreb $M_{1}, A B C$ and $G A$ index of crystal structure cubic carbon $C C C(n)$.

Fig.8: The comparison of $A B C_{4}$ and $G A_{5}$ index of crystal structure cubic carbon $C C C(n)$. 